\title{
Micromole per Hour per Millimole
}

National Cancer Institute

\section{Source}

National Cancer Institute. Micromole per Hour per Millimole. NCI Thesaurus. Code C124467.

A unit of fraction expressed as the ratio of the number of micromoles of substance per unit of time equal to one hour, to the amount of a different substance, in millimoles. 